\title{
Access to HIV services and viral load suppression among children during the 90-90-90 strategy implementation in South Africa: A time series analysis
}

\begin{tabular}{|c|c|}
\hline \multicolumn{2}{|c|}{$\begin{array}{l}\text { Authors: } \\
\text { Juliet C.Y. Nyasulu }{ }^{1,2} \\
\text { Innocent Maposa } \\
\text { Bernard P. Sikhakhane } \\
\text { Himani Pandya }{ }^{1} \text { (D) }\end{array}$} \\
\hline \multicolumn{2}{|c|}{$\begin{array}{l}\text { Affiliations: } \\
{ }^{1} \text { Division of Community } \\
\text { Paediatrics, Faculty of } \\
\text { Health Sciences, University } \\
\text { of the Witwatersrand, } \\
\text { Johannesburg, South Africa }\end{array}$} \\
\hline \multicolumn{2}{|c|}{$\begin{array}{l}{ }^{2} \text { Health Systems } \\
\text { Strengthening, AFRIQUIP, } \\
\text { Johannesburg, South Africa }\end{array}$} \\
\hline \multicolumn{2}{|c|}{$\begin{array}{l}{ }^{3} \text { Department of Epidemiology } \\
\text { and Biostatistics, Faculty of } \\
\text { Health Sciences, University } \\
\text { of the Witwatersrand, } \\
\text { Johannesburg, South Africa }\end{array}$} \\
\hline \multicolumn{2}{|c|}{$\begin{array}{l}{ }^{4} \text { Centre for HIV and STI's, } \\
\text { National Institute for } \\
\text { Communicable Diseases, } \\
\text { Johannesburg, South Africa }\end{array}$} \\
\hline \multicolumn{2}{|c|}{$\begin{array}{l}\text { 5JHB Health District: } \\
\text { Monitoring and Evaluation, } \\
\text { Gauteng Provincial } \\
\text { Department of Health, } \\
\text { Johannesburg, South Africa }\end{array}$} \\
\hline \multicolumn{2}{|c|}{$\begin{array}{l}\text { Corresponding author: } \\
\text { Juliet Nyasulu, } \\
\text { julietnyasulu@gmail.com }\end{array}$} \\
\hline \multicolumn{2}{|c|}{$\begin{array}{l}\text { Dates: } \\
\text { Received: } 29 \text { Oct. } 2020 \\
\text { Accepted: } 13 \text { Dec. } 2020 \\
\text { Published: } 17 \text { Mar. } 2021\end{array}$} \\
\hline \multicolumn{2}{|l|}{ Read online: } \\
\hline 回䗆回 & $\begin{array}{l}\text { Scan this QR } \\
\text { code with your } \\
\text { smart phone or } \\
\text { mobile device } \\
\text { to read online. }\end{array}$ \\
\hline
\end{tabular}

Background: During the era of the Millennium Development Goals (MDG), children were shown to have less access to human immunodeficiency virus (HIV) services than their adult counterparts; hence the call to prioritise children in the implementation of the Sustainable Development Goals (SDGs). However, South African (SA) national data in 2019 indicated that almost 3 years into the implementation of the $90-90-90$ strategy, only $59 \%$ of children living with HIV had been tested for HIV compared to $90 \%$ of adults.

Objectives: To evaluate the access of children to HIV services and record the viral load (VL) suppression rates during the implementation of the 90-90-90 strategy in the City of Johannesburg (COJ), South Africa.

Methods: This study applied a quasi-experimental interrupted time-series (ITS) design using the monthly District Health Information System (DHIS) and National Health Laboratory Services (NHLS) databases spanning the period from 2015 to 2020, that is, before and after the implementation and roll-out of the 90-90-90 strategy. Data were extracted from these databases into MS Excel 2010 spreadsheets and analysed with Stata 15 software from Stata Corp using a two-tailed $t$-test at a $5 \%$ level of significance.

Results: Overall, a significant increase was observed in the number of individuals tested for HIV, $n=757, p=0.0086$, and retained in care $n=2523, p=0.001$ over the whole period of analysis beginning in April 2015. Adult HIV testing, antiretroviral treatment (ART) initiation and retention in care had been decreasing in absolute numbers over a 10-month period before the intervention. An increase in these three data elements was observed following the implementation of the 90-90-90 program. On the other hand, children aged 0-15 years had demonstrated a significant increase in absolute numbers tested for HIV, $n=171, p=0.001$, but an insignificant increase in number of ART initiations, $n=14.33, p=0.252$, before implementation but a decrease after this. The overall VL suppression rates for children were lower than those of adults.

Conclusion: Although the COJ has recorded progress in adult HIV testing, ART initiation and retention, children living with HIV aged 0-15 years continue to experience less access to HIV services and lower VL suppression than youths and adults of $\geq 15$ years. Therefore, to ensure that the 90-90-90 targets are achieved across different age groups, children must be prioritised so that they can equally access these services with adults.

Keywords: 90-90-90 strategy; implementation; HIV testing; ART initiation; retention in HIV care; HIV care access by children.

\section{Introduction}

In the era of the Sustainable Development Goals (SDG's), the UNAIDS set countries, the ambitious '90-90-90' target of eradicating global infection with HIV by 2030. This required that by $2020,90 \%$ of people living with HIV would know their status, $90 \%$ of whom would be on antiretroviral treatment (ART) and 90\% of the latter, would be virally suppressed. ${ }^{1}$ The underlying principle behind these goals is the rapid scale-up of access to treatment for all PLWH, irrespective of their

How to cite this article: Nyasulu JCY, Maposa I, Sikhakhane BP, Pandya H. Access to HIV services and viral load suppression among children during the 90-90-90 strategy implementation in South Africa: A time series analysis. S Afr J HIV Med. 2021;22(1), a1187. https:// doi.org/10.4102/sajhivmed.v22i1.1187

Copyright: @ 2021. The Authors. Licensee: AOSIS. This work is licensed under the Creative Commons Attribution License. 
CD4 count. The primary target are cities as the risk of HIV and tuberculosis (TB) is greater in urban than rural areas. More than 200 cities and municipalities across the globe signed the United Nations declaration pledging commitment to these goals. ${ }^{1,2}$ Some progress was made: by 2018, 79\% (range, 67-92\%) of global PLWH knew their status. Of these, 78\% (range 69-82\%) were accessing ART and 86\% (range, 72-92\%) of the latter were virally suppressed. Targets for children and adolescents lagged behind. ${ }^{1}$

A total of 7.9 million South Africans are PLWH. Of these, \pm 4.9 million are on ART. ${ }^{3}$ The City of Johannesburg (COJ), together with 19 other South African (SA) cities and metropolitans, endorsed these 90-90-90 strategic goals. ${ }^{1,2}$ Whilst testing and treatment 'scale-up' in the COJ had been rapid in the preceding decade, the required 'doubling of effort' by 2020, could not be met. ${ }^{4}$ Nonetheless, progress had been made. Ninety per cent of SA-PLWH knew their status, $62 \%$ had been initiated on ART and 54\% were virally suppressed, that is, $90-62-54 \% .^{3,5}$ All was not good news, though. In 2019, only $63 \%$ of $0-15$-year-olds living with HIV were reported to be on ART. ${ }^{5}$ Compared with adults, HIV testing of children $<15$ years has been suboptimal. The 2019 SA-90-90-90 progress report indicated that only $59 \%$ of children living with HIV knew their status, and though $96 \%$ of those tested had been initiated onto ART, only $67 \%$ were virally suppressed; too few children are tested. ${ }^{6}$ This is despite the 2016-2030 SDG's strategy to prioritise children and the young. ${ }^{7,8}$ Although the national HIV statistics show a decline in the number of infected children and an increase in adults with HIV, the extent of the problem in the COJ has not been reported. ${ }^{3}$ This study evaluates the access of children $<15$ years to HIV services and the rates of viral load (VL) suppression during the implementation of the 90-90-90 strategy in the city.

\section{Methodology Study design}

This was a quasi-experimental study that aimed to establish the impact of the implementation of the 90-90-90 strategy on HIV testing, ART initiation, retention in care and viral suppression amongst PLWH in the COJ using an interrupted time series (ITS) analysis of data from the District Health Information System (DHIS) and the National Health Laboratory Service (NHLS). To strengthen the internal validity of the study, other factors that would impact HIV testing, ART usage and retention in care during the study period are explored and explained.

\section{Study participants}

The study included all individuals who tested positive for HIV and were initiated onto ART and who remained on ART in the COJ and on the DHIS database over the study period. In line with the DHIS age-categories, children were grouped into those $<59$ months of age and from 5 to $<15$ years. Those above 15 years were categorised as adults.

All VL tests performed by the NHLS during the study period were included.

\section{Study setting}

Data were collected from the COJ, Gauteng Province, South Africa. The city has seven regions (A-F), and a total population of \pm 5.6 million. This accounts for $36 \%$ of the Gauteng and $\pm 10 \%$ of the national population. ${ }^{3}$

One hundred and twenty-one primary healthcare (PHC) facilities offer HIV-services in the COJ and cater for \pm half-amillion PLWH. ${ }^{9}$

\section{The 90-90-90 strategy intervention}

The 90-90-90 strategy was conceptualised in 2015 and introduced to the COJ in January 2016. The district program implementation team identified indicators that would assist in attaining these targets. These indicators focused on the four pillars of HIV-care cascade, namely (1) prevention, (2) case identification, (3) treatment initiation, and (4) retention and success of treatment. Those indicators performing below target were described together with their root causes. Activities were proposed to address the gaps in the 90-90-90 district strategy implementation plan (DIP). ${ }^{7}$ Responsible personnel and the measures required to track progress were identified.

For example, one of the gaps in the district was poor access to services by youth and by men. As a result, social mobilisation and communication that targeted men and youth, key populations and vulnerable groups was implemented. The DIP was rolled out in January $2016 .^{7}$

\section{Data collection}

Data were collected from monthly reports of facilities captured in the DHIS database. All who were initiated on ART had a VL test - a measure of viral suppression and a proxy of adherence and retention in care - after 6 months and annually thereafter. More frequent VL monitoring is recommended where VL suppression is not achieved..$^{10}$ Viral suppression is defined as VL $<50$ copies / $\mathrm{mL}$ as per the 2019 South African National Department of Health (SANDoH) guidelines. ${ }^{10}$ The NHLS database provided viral suppression data. Data from the DHIS program was collated from April 2015 until March 2019 (48 months) and included the 9 months to January 2016 (before) and the 38 months after strategy implementation in January 2016. In addition, all VL suppression NHLS data from January 2015 to February 2020 (62 months) are included in this study. The proportion of virally suppressed 'test-results' has been calculated on a monthly basis throughout the 62 months for both adults and children.

\section{Outcome measures}

The study outcome measures included the number of individuals tested, initiated on ART, retained in care and virally suppressed in the COJ facilities before and after the January 2016 roll-out. 


\section{Statistical analysis}

Data preparation was done in MS Excel 2010 and analysed with Stata 15(Stata Corp). We used a two-tailed test at a 5\% level of significance throughout the analysis. Continuous data were expressed as the means (standard deviation, SD) or medians (interquartile range, IQR). Categorical data are presented as frequencies and percentages. The Durbin-Watson statistic was used to test for autocorrelation. We modelled the ITS data using segmented regression analysis which, as suggested by Wagner et al., has the advantage of adjusting for baseline level and trend, in this case, the HIV test-and-treat policy changes. ${ }^{11}$ For HIV testing, ART initiation and retention in HIV care, we compared the time series pattern 9 months before and after January 2016 when the 90-90-90 strategy implementation was rolled out in the COJ to assess differences. In addition, average monthly VL suppression rates from 12 months (January-December 2015) before were compared to 50 months (January 2016-February 2020) after strategy implementation roll-out. The trends before and after this intervention were analysed to assess whether the passage of time was associated with an increase or a decrease in the number of HIV tests, initiations and retention in care as well as viral suppression.

The specific model is: $Y_{t}=\beta_{0}+\beta_{1} X_{t}+\beta_{2}\left(I\left(t \geq t_{0}\right)\right)+\beta_{3} X_{t-t_{0}}+e_{t}$ where $Y_{t}$ is the total number of those HIV tested, ART initiated and remaining in care or VL suppression rate in a month in Johannesburg, which are response variables in this model; $X_{t}$ is a count time variable indicating time in months from the start of the observation period to intervention; intervention is an indicator $I\left(t \geq t_{0}\right)$ variable for time $t$ occurring before $\left(I\left(t \geq t_{0}\right)=0\right)$ or after $\left(I\left(t \geq t_{0}\right)=1\right) 90-90-90$ strategy roll-out, which was implemented at month $t_{0}$ in the series; and $X_{t-t_{0}}$ representing time after intervention is a count variable for the number of months after the intervention at time $t_{0}=10$ when assessing 90-90-90 indicators and $t_{0}=13$ when assessing suppression rates computed from NHLS data. Suppression was defined as having a VL below 50 copies/mL.

In this model, $\beta_{0}$ is the estimate of baseline level of the outcome, being the average number of patients tested for $\mathrm{HIV}$, initiated on ART and virally suppressed at time zero; $\beta_{1}$ estimates the mean change in total number or rate that occurred in each month before 90-90-90 strategy intervention; $\beta_{2}$ estimates the level of change in the average number of patients tested for HIV, initiated on ART and virally suppressed during the intervention month; and $\beta_{3}$ estimates the change in the trend in the mean monthly number of patients tested for HIV, initiated on ART and virally suppressed after the 90-90-90 strategy intervention implementation. The error term $e_{t}$ at time $t$ represents the random variability not accounted for by the model.

\section{Ethical considerations}

Ethical approval for this study was obtained on 01 October 2018 from the University of the Witwatersrand Human
Research Ethics Committee (Project research number: R14 / 49 and ethical clearance number: M180640).

\section{Results}

Numbers for HIV testing, ART initiation and those remaining in care before and after the implementation roll-out.

\section{Pre-intervention or pre-implementation}

All values are presented as medians with interquartile range [IQR].

The total number tested monthly for HIV in the preintervention period viz. April-December 2015 was a median of $n=64379$ (IQR, 58 425-66 374). Adults numbered 49650 (IQR, 47 644-52 092) of whom 6592 (IQR, 6328-6808) were in antenatal care (ANC). Under-5 years numbered 544 (IQR: $0-786$ ) and 5 to < 15-year-olds numbered 681 (IQR, 25-917).

Overall, the total number initiated monthly onto ART in the pre-intervention period was 4431 (IQR: 4375-4513). $N=101$ (IQR, 89-112) were children < 15 years. The number of adults was 4327 (IQR: 425-4388). The total number of PLWH retained in antiretroviral (ART) care monthly in the pre-intervention period was 256278 (IQR: 254 111-257 452). $N=10775$ (IQR, 10 704-10 832) were children $<15$ years of age. Youths and adults in care numbered 245509 (IQR: 243 392-246 620).

\section{Post-intervention or post-implementation}

All values are presented as medians with interquartile ranges [IQR].

The total number tested monthly for HIV during the 38 months post-intervention period was 75162 (IQR: 71 015-84 736). Youths and adults numbered 57027 (IQR, 51 831-64 825) of whom 7085 (IQR, 6725-7461) were in ANC care. The number of under-5 years was 1065 (IQR: 893-1194) and 5 to < 15 years was 1449 (IQR, 1174-2097) monthly.

The total number initiated monthly onto ART postintervention was 5288 (IQR, 4796-6053). $N=129$ (IQR: 103-173) were children $<15$ years. The youths and adults numbered 5134 (IQR: 4659-5925).

The total number of PLWH retained on ART monthly in the 38 months after intervention was 312360 (IQR, 284 296-338 008). The monthly numbers of children $<15$ years were 8688 (IQR, 8266-10 048). The median monthly number of all youths and adults retained in care was 303596 (IQR, 274 060-329 767).

\section{Number of viral load tests conducted before and} after intervention

A total of 2040018 VL tests were performed during the 62-month (5 years) period of the study on 23193 children 
TABLE 1: Number of viral loads captured annually in the City of Johannesburg before and after the intervention.

\begin{tabular}{|c|c|c|c|c|c|c|c|}
\hline Age category & $\begin{array}{l}\text { Pre-intervention } \\
\text { (ITV): 2015 } \\
\text { (12 months) }\end{array}$ & 2016 (post-ITV) & 2017 (post-ITV) & 2018 (post-ITV) & 2019 (post-ITV) & $\begin{array}{l}2020 \text { (post-ITV) Jan/ } \\
\text { Feb ( } 2 \text { months) }\end{array}$ & Total \\
\hline$<5$ years & 4575 & 4561 & 4667 & 4538 & 4191 & 661 & 23193 \\
\hline Adults & 264690 & 341270 & 396448 & 420527 & 455541 & 79786 & 1958262 \\
\hline Overall tests & 280221 & 357805 & 412698 & 436125 & 470991 & 82178 & 2040018 \\
\hline
\end{tabular}

ITV, intervention.

$<5$ years, 58563 children 5 to $<15$ years and 1958262 youths and adults (Table 1). Each newly diagnosed client had a VL at 6 and 12 months then annually thereafter and intermittently if clinically indicated. Follow-up tests number more than the total number of individual patients.

\section{HIV testing, antiretroviral treatment initiation and} retention in care before and after the intervention

\section{HIV testing before and after the intervention}

In the 10 months of 2015 that preceded the implementation of the 90-90-90 roll-out strategy, there was a non-significant monthly average decline in the number tested for HIV, $p=0.57$. However, categorised by age, HIV testing of adults did appear to decline significantly, $p<0.001$; whilst testing of children increased, 19 months to $<5$ years, $p=0.008$ and $\geq 5$ to $<15$ years, $p=0.001$ (Table 2, Item 1). After controlling for autocorrelation, at the time of the roll-out-strategy intervention in January 2016 (month 10) a non-significant, monthly-average increase of 1448 people tested for HIV, $p=0.27$ was recorded. Categorised by age, this testing showed a significant increase amongst adults $(p<0.001)$ and a significant decrease amongst children, 19 to $<5$ years, $p=0.008$, and those 5 to $<15$ years, $p=0.001$. Nevertheless, in the period 2015-2019, a significant overall average increase of 757 persons was recorded in the monthly average tested for $\mathrm{HIV}, p=0.009$.

\section{Post-intervention or post-implementation}

A non-significant increase in HIV testing was observed in the total adult group and the ANC attendees, though not significantly different from the baseline. On the other hand, significant declines in HIV testing amongst under-5 years ( $<59$ months) and 5 to $<15$ year groups were observed at the same time: $n=-101 ; p=0.021$ and $n=-171 ; p<0.001$, respectively.

\section{Overall trend throughout the study period}

As shown in Table 2, overall, there was a significant increase in HIV testing amongst adults and under-5 years children (< 59 months): $n=622 ; p=0.025$ and $n=14 ; p=0.002$, respectively, with no significant change from baseline for the 5 to $<15$-year-old group.

\section{Antiretroviral treatment initiation for those who are HIV positive}

Pre-implementation, the average numbers of monthly ART initiations amongst adults declined non-significantly by about 65 persons, $p=0.349$, but increased by \pm 14 persons amongst children $<15$ years, $p=0.252$. After implementation, an increase was observed in adults and a slight decrease in children $<15$ years.

Overall, the trend throughout the study period suggests an insignificant increase in ART initiation amongst adults and an insignificant decrease amongst children $<15$ years.

\section{Retention on antiretroviral treatment}

For 9 months before implementation, there was a nonsignificant total monthly average loss from HIV care of 462 PLWH, $p=0.515$. After January 2016, at the time of the strategy intervention, after controlling for autocorrelation, there was a monthly average increase of retention in care of 2985 PLWH, $p<0.001$. Overall, from 2015 to 2018, there was a significant monthly average increase of 2523 PLWH retained in ART care, $p<0.001$.

\section{Pre-intervention or pre-implementation}

As shown in Table 2, the average number of adults in ART care declined by about 418 persons, $p=0.536$, monthly and in children (the $<15$-year group) by \pm 49 persons monthly, $p=0.302$ (Table 2 ).

\section{Post-intervention or post-implementation}

There was an increase in adults and a decrease in children $<15$ years retained on ART care (Table 2 ).

\section{Overall trend throughout the study period}

There was a significant increase in numbers of adults on ART and a significant decrease in children $<15$ years.

\section{Overall HIV testing and antiretroviral treatment initiation trends over the study period}

Figure 1 shows the overall increasing monthly HIV testing trends over the study period and the consistent declines in update of HIV testing over the festive months of December and January.

Figure $2 \mathrm{a}$ and $2 \mathrm{~b}$ depicts the increasing numbers of those retained on ART amongst adults centrally to a continuous drop amongst children over the months. To note is the fact that adult and children retention in HIV care trends had some consistent drops during the festive season months of December to January. 
TABLE 2: The monthly average change in HIV testing and ART initiation of youths and adults ( $>15 y r s)$ and of children ( $<15 y r s)$ before ( 9 months) and after (38 months) the 90-90-90 implementation or intervention.

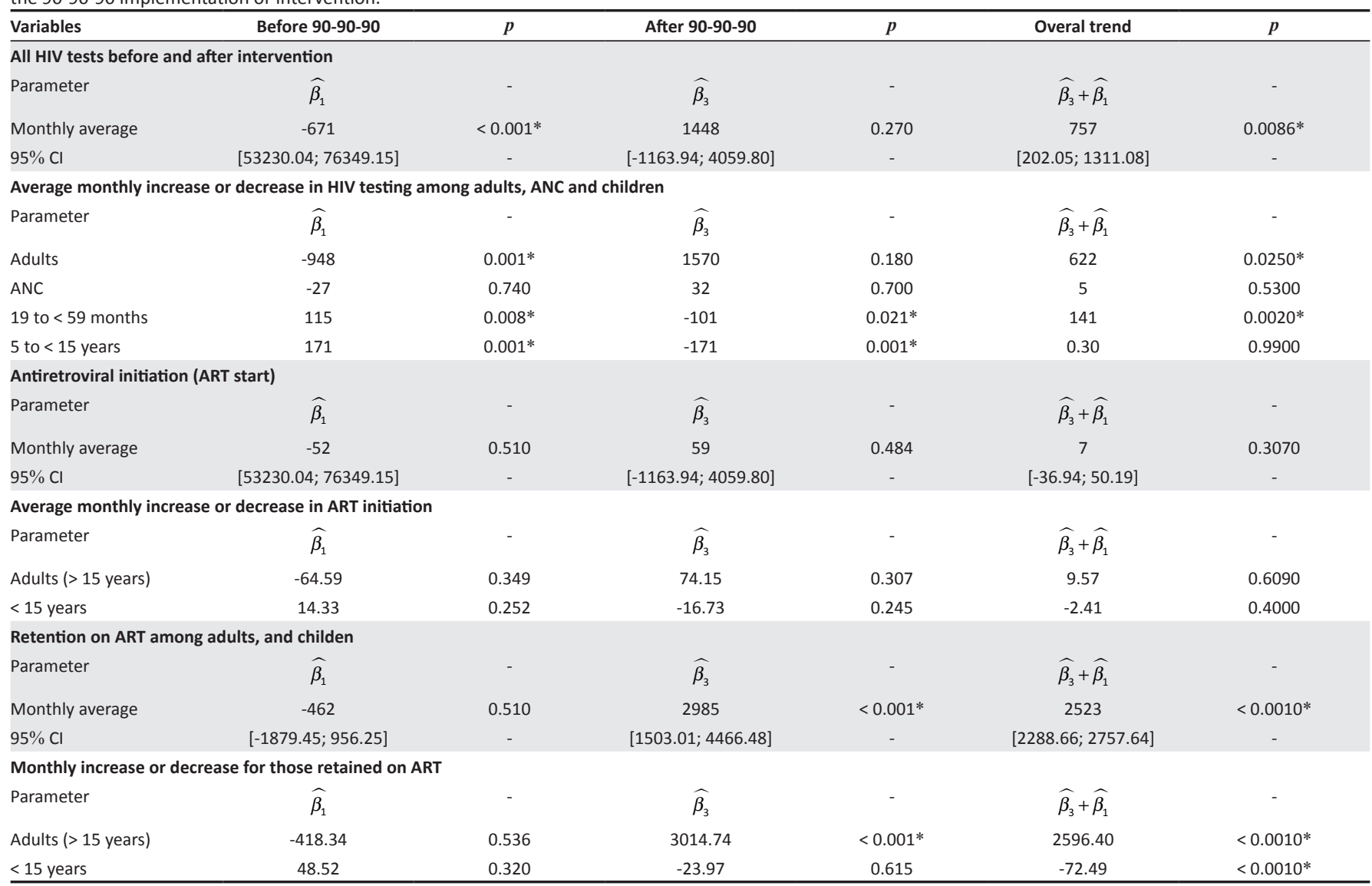

Note: *, Shows significant differences in the parameters before and after the strategy implementation. HIV, human immunodeficiency virus; ART, antiretroviral treatment; ANC, antenatal care; VL, viral load.

\section{Viral load suppression rates before and after the strategy roll-out}

As shown in Figure 3, the number of VL tests conducted during January 2015 to February 2020 indicates a higher suppression rate for adults compared to children through the 5 years pre- and post-implementation and roll-out. Throughout the period, a seasonal drop in VL suppression is observed between December and January of each year.

\section{Discussion}

This was a quasi-experimental study that utilised an ITS analysis to establish the effect of the 90-90-90 strategy implementation on HIV testing, ART initiation and retention in care amongst HIV positive individuals in the COJ. Overall, a significant increase was observed in the number of individuals tested for HIV, initiated on ART and retained in HIV care after the implementation. Adult HIV testing, ART initiation and retention in HIV care had decreased significantly before implementation but was followed by a significant increase over time in all three aspects once the strategy was rolled out. On the other hand, all three measures in children aged $0-15$ years had been increasing before implementation but decreased significantly thereafter.

Throughout the study period, rates of viral suppression were less in children than in adults, though trends were similar
(Table 3). Rates of HIV testing, ART initiation, retention in care and VL suppression declined in December and January each 'festive season' - throughout the study period.

\section{HIV testing, antiretroviral treatment initiation and retention in care for adults}

Our data indicate that the implementation of the 90-90-09 strategy in the COJ has had a positive effect amongst adults accessing HIV services in the city. Other studies support this assessment. ${ }^{12,13}$ The roll-out of 'Universal Test and Treat' (UTT) in September 2016 would have contributed to this outcome as this removed the CD4 count 'barrier' to care. ${ }^{14,15}$ Furthermore, the roll-out of the 90-90-90 strategy was accompanied by HIV testing campaigns that raised awareness in the community. ${ }^{7}$

\section{HIV testing, antiretroviral treatment initiation and retention in care for $\mathbf{0 - 1 5}$ year-olds}

A concern is the apparent decline of HIV testing amongst children aged $0-15$ years. This followed an increase in testing in the pre-implementation phase. The most recent SANDoH implementation progress-report noted that only $\pm 59 \%$ of children living with HIV (CLWH) are being diagnosed. Although $96 \%$ of those who test positive are initiated onto ART, only $66 \%$ are retained in care. In reality, this means that only about $38 \%$ of CLWH achieve retention on ART. ${ }^{6}$ 
Furthermore, the HIV screening strategy amongst the 0-15 year-olds during the study period missed a large number of CLWH, especially children infected postnatally via breastfeeding practices, or perinatally with delayed seroconversion post-delivery. ${ }^{16}$ Birth PCR tests on all exposed infants are now helping to close this gap. ${ }^{17,18}$ Additional failures occur during or at the end of breastfeeding, or when birth results are not checked and when follow-up and linkage to care are not established. ${ }^{19}$ Point-of-care infant testing may assist in the prevention of some gaps. ${ }^{20}$ Current South African infant-testing guidelines recommend testing at birth, 10 weeks and upon ending breastfeeding. ${ }^{14}$ Nonetheless, data from Tshwane district report that only a third of HIV-exposed children repeat the HIV test at 18-24 months and only a quarter at the end of breastfeeding. ${ }^{21}$ Although early infant diagnosis using PCR at birth and 10 weeks has impacted infant HIV testing, ${ }^{22}$ gaps remain. Consequently, the current testing of children is suboptimal compared to adults. ${ }^{6}$ The system is failing children. Greater effort must be made to trace and test exposed children, especially post-breastfeeding as these 18-month rates are extremely low. ${ }^{6,23}$

Our study found that ART initiation rates in $<5$-year-old children have declined. In part, this results from the country's

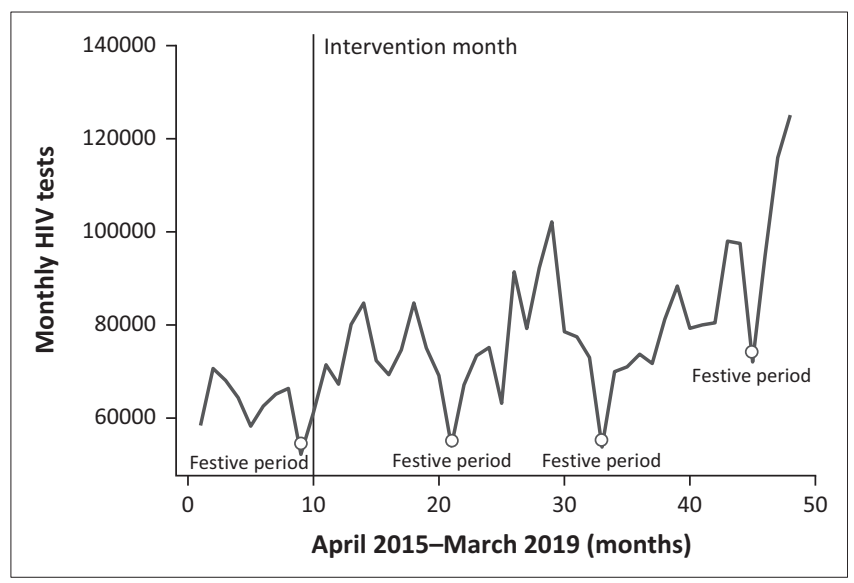

FIGURE 1: Overall average monthly increase or decrease in human immunodeficiency virus testing in the City of Johannesburg. excellent prevention of mother-to-child transmission of HIV (PMTCT) rate of $0.9 \%$ and the COJ's $1 \%{ }^{24}$ Fewer children are now needing to be initiated on ART. However, our study also found that fewer children are being tested. Gaps and opportunities are being missed. ${ }^{6}$ Innovative approaches are still needed in support of this population group. ${ }^{20,25}$

The testing, initiation and retention in care data of the 5 to $<15$-year-old cohort changed from an increase in 2015 to a significant decline after the implementation of the 90-90-90 strategy. A possible explanation is that at the end of the implementation phase of the Millennium Development Goals (MDG) goals (2014-2015), it was clear that children were being missed and a special call was made to reverse this situation with the implementation of the SDGs. A concerted effort was made to prioritise HIV testing and ART initiation in this age group. ${ }^{8}$ Although the COJ had a 2016-2020 DIP which covered activities to reach youths, it did not prioritise those aged $<15$ years. ${ }^{8,26}$ Reports in the SDG implementation era still document the inadequate provision of services to children and youth; something that must be rectified in the $2020 / 2030$ targets. ${ }^{13,23}$

Concerning children and youths, the NHLS database indicated that throughout the reporting period, the younger

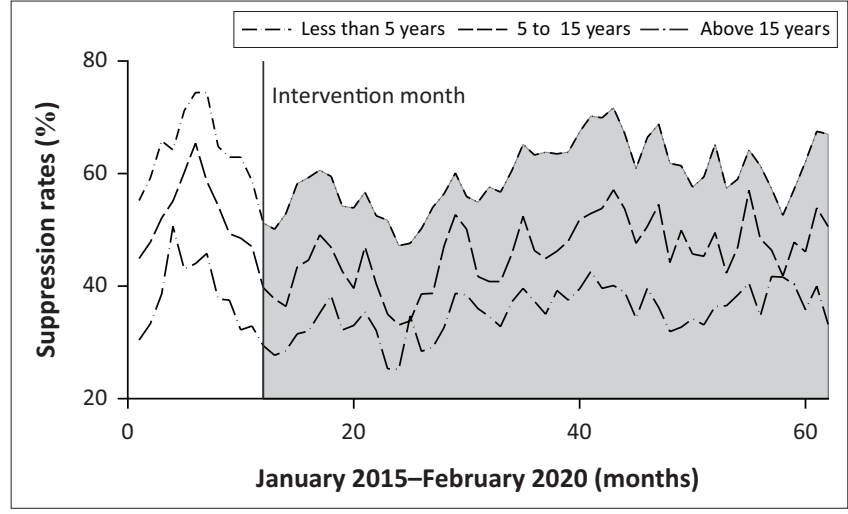

FIGURE 3: Proportions of those virally suppressed before and after the 90-90-90 strategy implementation.

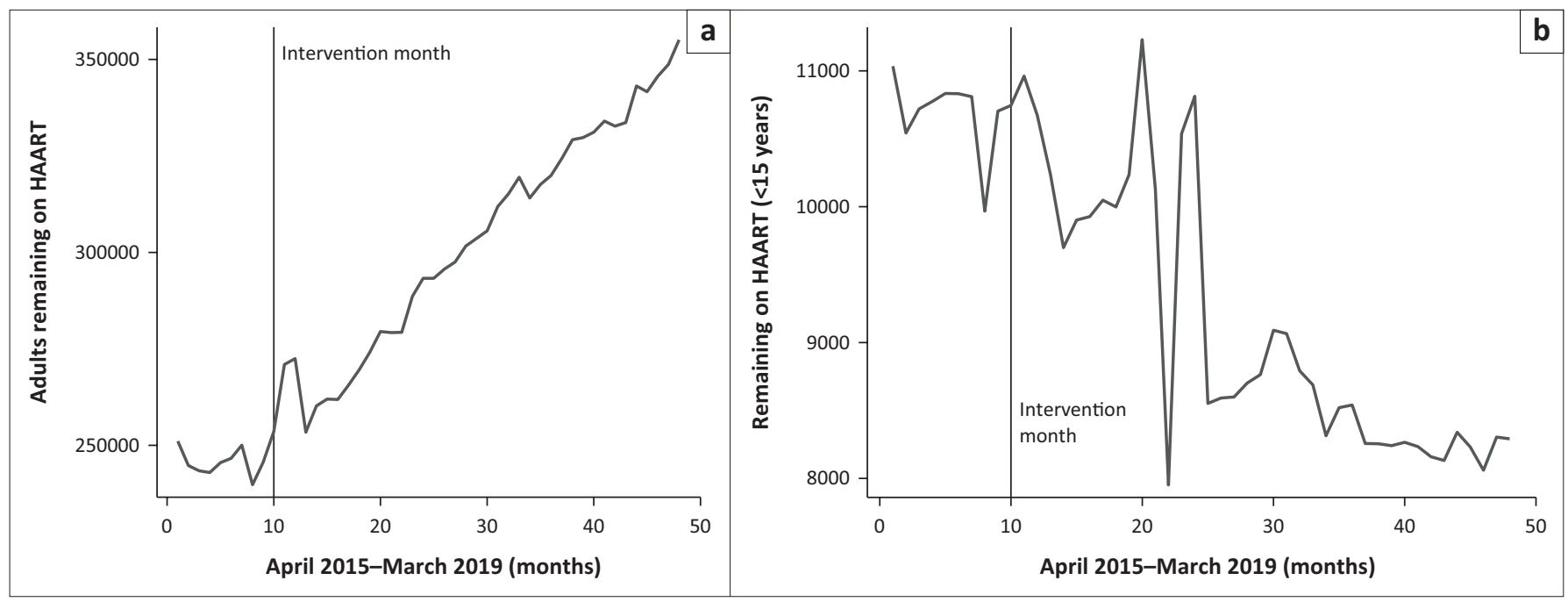

FIGURE 2: (a) Trends in adults monthly retention on antiretroviral treatment and (b) trends in $<15$ years monthly retention on antiretroviral treatment. 
TABLE 3: Viral load suppression rate before and after the $90-90-90$ strategy implementation amongst children and adults. Viral load defined at $\mathrm{VL}<50$ copies $/ \mathrm{mL}$

\begin{tabular}{lcccc}
\hline Intervention trends & Parameter & Adults & $\begin{array}{c}\text { Under } \\
\text { 5 years }\end{array}$ & $\begin{array}{c}\mathbf{5 - 1 5} \\
\text { years }\end{array}$ \\
\hline Before 90-90-90 strategy & $\widehat{\beta_{1}}$ & -0.32 & -0.009 & 0.04 \\
$p$ & & 0.645 & 0.990 & 0.953 \\
Change at intervention & $\widehat{\beta_{2}}$ & -9.1 & $-6 \%$ & -10 \\
$p$ & & 0.002 & 0.1 & 0.002 \\
After 90-90-90 strategy & $\widehat{\beta_{3}}$ & -0.1 & +0.15 & +0.15 \\
$p$ & & 0.900 & 0.800 & 0.800 \\
Overall trend & $\widehat{\beta_{3}}+\widehat{\beta_{1}}$ & 0.202 & 0.139 & 0.187 \\
$p$ & & 0.080 & 0.014 & 0.033
\end{tabular}

Note: A drop in the VL suppression rate was observed over the 12-month observation period before the intervention in both children and adults in 2015. This drop was significan amongst adults and children aged 5-15 years. This was followed by a non-significant and slow rise in children's viral suppression rates and a drop amongst adults. Overall, only children aged $<5$ years and 5 to $<15$ years showed a marginal but significant increase in VL suppression over the 5 -year study period.

the individual, the lower the VL suppression rate. Whilst children aged $<5$ years and 5 to $<15$ years showed a small but significant increase in the overall VL suppression over the 5-year study period, this was still below that of the adults. The suboptimal performance of children triangulates with the DHIS database and is similar to other studies. ${ }^{6}$ Innovative solutions are needed to assist in ensuring that more children are tested, initiated and adhere to ART. Five years into the implementation of SDG goals, our children lag behind their adult counterparts. Urgent action is called for.

Of interest is the 'lapse' in the performance indicators across all age groups in the December to January festive period. Not much has been documented regarding patients' access and adherence to care during this time.

However, we speculate that the lower rates of performance at these times reflect the annual migration of city-dwellers to ancestral homes in South and Southern Africa - appointments are missed and treatment adherence impaired and VL suppression rates fall. ${ }^{3}$ It is critical to establish whether this is the case and what mechanisms are needed to mitigate the problem. For example, the provision of more than one repeat of medication to cover the holiday duration. And the scheduling of the COJ's HIV testing campaigns to avoid the holiday seasons.

Study limitations include the retrospective nature of the data and our inability therefore to link cause and effect.

Caution is suggested when interpreting sensitivity data where numbers of our children analysed were extremely low. The study also records that the COJ was not able to fulfil the UNAIDS 90-90-90 target by 2020. The databases and data recorded in healthcare facilities were at times incomplete. However, we applied quality tracing to our resources and are confident that the outcome in this study reflects that data. The strength of the study is in the large cohort size and the reproducibility and integrity of the data over a prolonged 5-year assessment period.

\section{Conclusion}

This study highlights the suboptimal level of access to HIV services and VL suppression of children compared to adults during the 90-90-90 strategy implementation in the COJ. Serious consideration is recommended to ensure that children and their guardians enjoy equity with adults in accessing healthcare services in the COJ if 90-90-90 targets are to be met. Plans must be implemented to mitigate the loss of HIV care and control that appears to be occurring during the holiday season.

\section{Acknowledgements}

The authors would like to acknowledge the support from the City of Johannesburg team for provision of data and information on the 90-90-90 strategy implementation progress. In addition, the help from Prince Dulaze of ANOVA Health, the implementation partner, is also greatly appreciated.

\section{Competing interests}

The authors declare that they have no financial or personal relationships that may have inappropriately influenced them in writing this research article.

\section{Authors' contributions}

J.C.Y.N. conceptualised the research project and drafted the first version of this article. I.M. and B.P.S. analysed the data and drafted the results section. J.C.Y.N., I.M., B.P.S. and H.P. read, revised the drafts and approved the final manuscript. J.C.Y.N. is the guarantor of the manuscript.

\section{Funding information}

The authors declare that they have no financial or personal relationships that may have inappropriately influenced them in writing this article.

\section{Data availability}

Data analysed for this study will be made available when the need arises.

\section{Disclaimer}

The views and opinions expressed in this article are those of the authors and do not necessarily reflect the official policy or position of any affiliated agency of the authors.

\section{References}

1. UNAIDS global AIDS update 2019. Communities at the centre: Defending rights, breaking barriers, reaching people with HIV services [homepage on the Internet]. 2019 [cited 2020 Sept 1]. Available from: https://www.unaids.org/sites/default/ files/media_asset/2019-global-AIDS-update_en.pdf

2. UN political declaration on HIV and AIDS. On the fast track to accelerating the fight against HIV and to ending the AIDS epidemic by 2030. Geneva: United Nations; 2019.

3. SA Statistical Release. Midyear population estimates. Department of Statistics South Africa, editor. Pretoria: ISIbalo House; 2019.

4. Stuart M, Fraser-Hurt N, Mabusela E, et al. The City of Johannesburg can end AIDS by 2030: Modelling the impact of achieving the Fast-Track targets and what it will take to get there. J Int AIDS Soc. 2018;21(1):e25068. https://doi.org/10.1002/jia2.25068 
5. UNAIDS. Ending the AIDS epidemic by 2030, Program area: South Africa [homepage on the Internet]. 2019 [cited 2020 July 5]. Available from: https:// www.unaids.org/en/regionscountries/countries/southafrica

6. Pillay Y, editor. Setting the scene: Some data on the HIV epidemic. 9th SA AIDS Conference; 2019 June 11-14; Durban: SA AIDS Conference; 2019.

7. Health Systems Trust. City of Johannesburg district implementation plan (DIP). In CoJ. 90-90-90 strategy district implementation plan. Volume 1. Durban: Health Systems Trust; 2016 (Unpublished).

8. UNAIDS. How AIDS changed everything - MDG 6: 15 years, 15 lessons of hope from the AIDS response to all who have worked to achieve the millennium development goals. UNAIDS, editor. Geneva: UNAIDS; 2015

9. IHME. Local burden of disease - HIV. Viz Hub [homepage on the Internet]. 2018. Available from: https://vizhub.healthdata.org/lbd/hiv

10. SA. 2019 ART clinical guidelines for the management of HIV in adults, pregnancy, adolescents, children, infants and neonates. Republic of South Africa National Department of Health, editor. Pretoria: Republic of South Africa National Department of Health; 2019.

11. Wagner A, Soumerai S, Zhang F, Ross-Degnan D. Segmented regression analysis of interrupted time series studies in medication use research. J Clin Pharm Therap. 2002;27(4):299-309. https://doi.org/10.1046/j.1365-2710.2002.00430.x

12. Lippman S, Ayadi E, Grignon A, et al. Improvements in the South African HIV care cascade: Findings on 90-90-90 targets from successive population-representative org/10.1002/jia2.25295

13. Huerga $H$, Van Cutsem G, Puren A, et al. Progress towards the UNAIDS 90-90-90 goals by age and gender in a rural area of KwaZulu-Natal, South Africa: A household-based community cross-sectional survey. BioMed Cent Public Health 2018;18(1):303. https://doi.org/10.1186/s12889-018-5208-0

14. SA. National consolidated guidelines for the prevention of mother-to-child transmission of HIV (PMTCT) and the management of HIV in children, adolescents and adults. Health, editor. Pretoria: NDOH, 2015; p. 136

15. Nyasulu J. Pre-ART program service delivery at a PHC facility level: Access and retention of patients in care in the city of Johannesburg, South Afric [homepage on the Internet]. [Monograph]. Johannesburg: University of the Witwatersrand; 2016 [cited 2020 July 10]. Available from: http://wiredspace. wits.ac.za/handle/10539/21246

16. Johnson L, Dorrington R, Moolla H. Progress towards the 2020 targets for HIV diagnosis and antiretroviral treatment in South Africa. S Afr Med J. 2017;18(1):a694. https://doi.org/10.4102/sajhivmed.v18i1.694
17. Wessels J, Sherman G, Bamford L, et al. The updated South African national guideline for the prevention of mother to child transmission of communicable infections (2019). South Afr J HIV Med. 2020;21(1):1079. https://doi.org/10.4102/ infections (2019). South

18. Mnyani C, Tait $\mathrm{CL}$, Peters RPH, et al. Implementation of a PMTCT programme in a high HIV prevalence setting in Johannesburg, South Africa: 2002-2015. S Afr J HIV Med. 2020;21(1):a1024. https://doi.org/10.4102/sajhivmed. v21i1.1024

19. Phiri NA, Lee HY, Chilenga L, et al. Early infant diagnosis and outcomes in HIV exposed infants at a central and a district hospital, Northern Malawi. Public Health Action. 2017;7(2):83-89. https://doi.org/10.5588/pha.16.0119

20. Spooner E, Govender K, Reddy T, et al. Point-of-care HIV testing best practice for early infant diagnosis: An implementation study. BMC Public Health. 2019;19(1):731. https://doi.org/10.1186/s12889-019-6990-z

21. Moloko SM. Factors associated with the HIV transmission rate in 18 to 24 month-old children enrolled in the prevention of mother-to-child transmission programme at the City of Tshwane clinics [homepage on the Internet]. Pretoria: University of South Africa [cited 2020 Aug 14]. Available from: http:// hdl.handle.net/10500/13817

22. Technau K, Kuhn L, Coovadia A, Carmona S, Sherman G. Improving early identification of HIV-infected neonates with birth PCR testing in a large urban hospital in Johannesburg, South Africa: Successes and challenges. J Int AIDS Soc. 2017;20(1):21436. https://doi.org/10.7448/IAS.20.01/21436

23. Davies M, Pinto J. Targeting 90-90-90 - Don't leave children and adolescents behind. J Int AIDS Soc. 2015;18(Suppl 6):20745. https://doi.org/10.7448/ IAS.18.7.20745

24. Goga A, Chirinda W, Ngandu N, et al. Closing the gaps to eliminate mother-to-child transmission (MTCT) of HIV in South Africa: Understanding MTCT case rates, factors that hinder the monitoring and attainment of targets, and potential game-
changers. S Afr Med J. 2018;108(3 Suppl 1):S17-S24. https://doi.org/10.7196/ SAMJ.2017.v108i3b.12817

25. Kellerman S, Essajee S. HIV testing for children in resource-limited settings: What are we waiting for? PLoS Med. 2010;7(7):e1000285. https://doi.org/10.1371/ journal.pmed.1000285

26. Sherman G, Lilian R, Bhardwaj S, Candy S, Barron P. Laboratory information system data demonstrate successful implementation of the prevention of mother-tochild transmission programme in South Africa. S Afr Med J. 2014;104(Suppl. 1): 235-258. https://doi.org/10.7196/SAMJ.7598 